\title{
Experimental models in vaccine research: malaria and leishmaniasis
}

\author{
C. Teixeira and R. Gomes \\ Centro de Pesquisas Gonçalo Moniz, Fundação Oswaldo Cruz (FIOCRUZ), Salvador, BA, Brasil
}

\begin{abstract}
Animal models have a long history of being useful tools, not only to test and select vaccines, but also to help understand the elaborate details of the immune response that follows infection. Different models have been extensively used to investigate putative immunological correlates of protection against parasitic diseases that are important to reach a successful vaccine. The greatest challenge has been the improvement and adaptation of these models to reflect the reality of human disease and the screening of vaccine candidates capable of overcoming the challenge of natural transmission. This review will discuss the advantages and challenges of using experimental animal models for vaccine development and how the knowledge achieved can be extrapolated to human disease by looking into two important parasitic diseases: malaria and leishmaniasis.
\end{abstract}

Key words: Human vaccines; Animal models; Malaria; Leishmaniasis

\section{Introduction}

The use and development of experimental models has been closely related to the history of vaccine research. The advantages of exploring experimental animal models include some aspects that cannot be considered in clinical trials. The animals are easily available and affordable, allowing the use of a significant number of subjects and the evaluation of numerous and diverse potential antigen candidates. Moreover, they are suitable to investigate disease progression and to analyze virulence factors of particular isolates or modified strains of parasites.

Among the different animals that have been used as models for scientific research, rodents have been used the most, especially for the numerous advantages that they offer. Concerning immunological investigations, the availability of inbred, knockout and transgenic mice that can be used to investigate specific cells and molecules of the immune system have made mice a rich source for understanding the host immune response. Mice can also be used for the evaluation of vaccine immunity and longterm immune memory, which cannot be obtained from people living in endemic areas where re-exposure or other factors are not controlled. Organs and tissues where parasites may survive or be sequestered can be easily accessed for a more accurate evaluation of the disease.

Despite the numerous advantages offered by experimental models, there are some important limitations that should always be taken into consideration. The major challenge to establish a good experimental model is the capacity of extrapolating the findings to human disease. Human populations have a diverse genetic background that has a profound influence on the immune response, while most animal models are usually based on inbred strains corresponding to a homogeneous genetic population. Data resulting from experimental models can be consistent and relevant but may not reflect how some individuals can develop disease or may remain naturally resistant.

This limitation can be solved by the use of outbreed large animal models that are more closely related to humans, like dogs and non-human primates. This characteristic makes them a useful model for investigating infectious diseases where the pathological and clinical alterations would mimic the human response. Nevertheless, in spite of the striking similarities, some differences such as the more diverse histocompatibility complex and absent HLA-A2 in chimpanzees can be sufficient to alter the disease pattern and the immune response. In addition, the cost of large animals can be high and, for some species, important immunological tools are missing, which limits the complete characterization of the host immune response $(1,2)$.

Although there are limitations, the use of animal models, particularly regarding the relevance of the results to human trials and gaps in the knowledge of the biology

Correspondence: R. Gomes, Centro de Pesquisas Gonçalo Moniz, Fundação Oswaldo Cruz (FIOCRUZ), Rua Waldemar Falcão, 121, 40296-710 Salvador, BA, Brasil. E-mail: rbrandim@gmail.com 
of the host-pathogen interaction, it is important that any vaccine be tested in animal models to assess its immunogenicity, safety and efficacy. Here, we consider the knowledge acquired by using experimental models in the search for a vaccine against two major parasitic diseases: malaria and leishmaniasis.

\section{Experimental models used to test vaccines against malaria}

Malaria is a devastating disease that affects about 500 million people worldwide, mostly children under 5 years of age. It is caused by Plasmodium spp and is transmitted by the bite of infected Anopheles mosquitoes. Following inoculation of sporozoites by infected mosquitoes the parasites quickly migrate and invade hepatocytes, where they multiply and later transform into merozoites. These forms are released from the infected cells and invade erythrocytes, initiating a cycle that leads to repeated parasite multiplication causing severe anemia or cerebral malaria (3).

Malaria is one of the most devastating parasitic diseases largely due to the absence of effective vaccines and the appearance of drug-resistant strains. Protection of mice, non-human primates and humans following vaccination with irradiated sporozoites has demonstrated the feasibility of developing a universally effective, longlasting vaccine (4-7).

Experimental models were used from the beginning in malaria studies and have provided important insights into the mechanism of Plasmodium spp pathogenesis $(8,9)$. Several avian models were used in early studies to screen for new potential treatments and candidate vaccines, and to help understand parasite biology. However, avian models proved to be a poor alternative for studies with mammalian hosts. The establishment of a rodent malaria model with $P$. berghei opened a new perspective and brought new insights into malaria infection in mammals (10). Other species, like $P$. chabaudi, $P$. yoelii, and $P$. vinckei, were also shown to naturally infect mice and to provide models with different disease outcomes and levels of susceptibility in inbred mouse strains (11).

Mouse models have helped elucidate the mechanisms implicated in the protective immune response. Due to the complex life cycle of the parasite, the host immune response to infection is varied, with both humoral and cellmediated immune responses involved in eliciting effective immunity. For instance, early reports based on murine models established that $\mathrm{T}$ cells are important for the immune response against the liver stage, showing that $T$ cells are initially directed at the intrahepatic parasite. Within the $\mathrm{T}$ cell population, $\mathrm{CD}^{+} \mathrm{T}$ cells play an important role in protection against rodent malaria, especially in $P$. berghei and $P$. yoelii infections, by inhibiting parasite growth in the liver. $\mathrm{CD}^{+} \mathrm{T}$ cells also play an important role in the pre-erythrocytic stages of rodent malaria as either helper or effector cells (12-15).
Although experimental models have been useful tools for dissecting the immune response, there is no concrete evidence indicating that the same mechanism takes place in human malaria.

Unfortunately, there is no reliable animal model for human malaria other than non-human primates. Nonhuman primates represent a valuable resource for testing vaccine candidates and drugs for human use prior to human clinical trials. The Aotus genus, largely used in malaria research, is not found naturally infected with human malaria parasites but represents a valuable model to test vaccines. Aotus can be easily infected with $P$. falciparum and $P$. vivax with different levels of susceptibility, depending on the combination of Aotus and parasite species. For instance, protection has been achieved in $A$. nancymai immunized with the $P$. falciparum merozoite surface protein 1 antigen against blood-stage challenge with $P$. falciparum (16). Rhesus macaques have also been used as models to assess cerebral malaria, an important clinical aspect when considering a vaccine against $P$. falciparum (17).

Although mice and non-human primates offer many advantages for the study of malaria, some limitations should be considered. The lack of immunological tools to assess the immune response of non-human primates and the poor translation of antigens that are protective in mouse models to human malaria indicate that there is an urgent need to improve the experimental models. In an attempt to circumvent these issues, researchers have engineered murine models to mimic human immune responses, such as mice that are transgenic for human molecules or receptors $(18,19)$. Recent studies have also highlighted the importance of natural transmission by infected mosquitoes to test vaccine candidates as a more reliable form of challenge. The response of mice challenged with $P$. berghei- or $P$. chabaudi-infected mosquito bites confirmed that the natural transmission method is the most relevant challenge regarding the testing of vaccines in animal models $(20,21)$.

\section{Experimental models for testing vaccines in leishmaniasis}

Leishmaniasis is a group of diseases caused by protozoa of the genus Leishmania, causing significant morbidity and mortality worldwide. The disease is endemic in 88 countries and, according to the World Health Organization, 350 million people are at risk of infection with a worldwide prevalence of 12 million cases. Different clinical manifestations (cutaneous, mucocutaneous, diffuse cutaneous, and visceral) are caused by different species of Leishmania and the genetic background of the host can markedly influence the outcome of infection. The infection begins when an infected female sand fly inoculates Leishmania parasites into the skin of a vertebrate host $(22,23)$. The sand fly regurgitates the parasite and promastigote secretory gel (PSG) while 
injecting saliva $(24,25)$. In the skin, the parasite penetrates mainly macrophages, where they multiply transformed into amastigotes.

To date, there is no global vaccine able to prevent leishmaniasis but vaccine research has advanced in recent years. These advances are mostly due to improved mouse and large animal models (such as dogs and monkeys), which have increased the knowledge of the immunological mechanisms involving the control of infection $(26,27)$. However, it is important to state that all the information generated using these models does not automatically extrapolate to the disease in humans $(22,28)$. More recently, the introduction of certain elements, such as low parasite inoculum, co-injection with sand fly saliva and PSG, site of inoculation, and challenge using a live infected sand fly, have been able to improve animal models in reflecting the course of natural infection (29).

\section{Cutaneous leishmaniasis}

Cutaneous leishmaniasis (CL) is described as a localized skin lesion that develops in the same area where the infected sand fly had previously fed. Depending on various factors like parasite species, immunological status and genetic background of the host, the lesion can be self-healing or may require treatment. The infection of mice with Leishmania major is one of the best studied leishmaniasis models, with the mouse developing aspects similar to those of the human disease (27). The conventional $L$. major mouse model injects a high dose of parasites into a subcutaneous site. BALB/c mice are highly susceptible to $L$. major and develop uncontrolled lesions. In contrast, C57BL/6 mice are resistant to $L$. major infection, developing small lesions that heal by about 12 weeks after infection, a fact that correlates with human disease (30).

In mice, both susceptible and resistant profiles show CD4+ $\mathrm{T}$ cell subset differentiation in vivo. L. major infection leads to the development of a polarized Th1 or Th2 immune response that dictates resistance or susceptibility, respectively. The Th1 cytokines activate macrophages to kill the intracellular parasite, primarily through a nitric oxide-mediated mechanism (31). The importance of the Th1/Th2 balance in the regulation of disease outcome, in vivo, was demonstrated in several studies, which have stressed that a successful vaccine against CL must induce the differentiation and expansion of specific CD4+ Th1 lymphocytes $(22,32)$.

In experimental sand fly transmission, the estimated number of metacyclic promastigotes inoculated by the infected sand fly into the skin revealed a wide variation in the number of parasites delivered (30). During transmission, sand fly saliva and PSG are also co-injected into the skin and were shown to exacerbate lesion development $(24,25)$. Titus and Ribeiro (24) first demonstrated that saliva was able to enhance Leishmania infection. This effect was observed when different species of Leishmania (L. major, L. braziliensis and L. amazonensis) were coinjected with salivary gland sonicate (SGS) from two vector species (Lutzomyia longipalpis and Phlebotomus papatasi) (33-35). The exacerbative effect of co-injecting salivary molecules was later related to potent molecules present in the saliva that modulate the balance of Th1/Th2 to a response that is favorable to parasite establishment and survival (36-38).

Since then, research has focused on trying to adapt experimental models to incorporate sand fly saliva. Belkaid et al. (26) sought to develop an animal model of cutaneous leishmaniasis that could mimic the natural conditions of Leishmania infection. In their study, a small quantity of $L$. major (1000 metacyclic promastigotes) coinjected with SGS obtained from a natural vector, $P$. papatasi, was injected into the mouse ear dermis. This resulted in a dramatic exacerbative effect on lesion development in both BALB/C and $\mathrm{C} 57 \mathrm{BL} / 6$ mice. This model was used to test a vaccine based on a singlesalivary molecule, a DNA vaccine expressing a $15-\mathrm{kDa}$ protein from $P$. papatasi saliva that was able to confer protection in vaccinated mice challenged with $L$. major plus SGS (39). The vaccination also provided protection in B-cell knockout mice, indicating that a delayed-type hypersensitivity (DTH) generated against sand fly saliva was responsible for most or all the protective effects of this vaccine and that molecules present in the saliva are important targets for controlling leishmaniasis. Interestingly, mice vaccinated with maxadilan, a salivary molecule from $L$. longipalpis saliva, were also protected against challenge with $L$. major co-injected with SGS (40). These results show that sand fly salivary gland components are also potent immunogenic molecules, reinforcing the importance of including sand fly saliva as a component for an anti-Leishmania vaccine.

Although the model of injecting saliva with the parasite into the skin brought new perspectives in conditions that mimic a natural infection, it was later adapted to a model of natural transmission with sand flies where all the elements (saliva, parasite and PSG) are present. This natural model demonstrated that it is possible to transmit $L$. major by the bite of its natural vector, P. papatasi, to $\mathrm{BALB} / \mathrm{c}$ and $\mathrm{C} 57 \mathrm{BL} / 6$ mice. Moreover, pre-exposure to uninfected bites resulted in a significant reduction in lesion pathology when compared with naive mice. The protection observed in mice pre-exposed to saliva, either by needle inoculation or by uninfected sand fly bites, involved a strong DTH reaction against saliva $(26,41)$. In mice sensitized by bites, protection was associated with a strong upregulation of IFN- $\gamma$ and IL-12 at the bite site, indicating the activation of macrophages to kill parasites and suggesting the acceleration of anti-Leishmania immunity.

The natural transmission model has been used in recent work by challenging mice with infected sand flies, 
demonstrating the relevance of testing potential vaccine candidates using infected sand flies. The natural challenge model was first verified when mice vaccinated with glycoconjugates derived from PSG were protected against challenge against infected sand flies, but mice vaccinated with another antigen preparation, a Leishmania antigen plus IL-12 that was effective against needle challenge, had no protection against infected sand fly bites (42). In agreement with these findings, it was demonstrated that mice vaccinated with a killed vaccine comprised of an autoclaved L. major antigen and CpG oligodeoxynucleotides that conferred protection against needle challenge with parasites, failed to protect against infected sand fly challenge (43). More recently, it was demonstrated that two vaccine candidates, comprised of a Leishmania (KSAC) and a salivary gland antigen (LJM11), were able to control Leishmania infection following challenge with infected sand flies $(32,44)$.

These recent findings emphasize the critical role that the sand fly plays in parasite transmission and how the adaptation of experimental models to this natural challenge should be considered in future vaccine development studies.

\section{Visceral leishmaniasis}

Visceral leishmaniasis (VL), the most severe form of leishmaniasis, is caused by parasites of the Leishmania donovani complex ( $L$. donovani and $L$. infantum). VL severely compromises the spleen, liver and bone marrow of the host leading to fever, hepatosplenomegaly, pancytopenia, and cachexia and is fatal if not treated.

Different animal models, especially mice and hamsters, have been used and adapted to mimic human VL and to test vaccine candidates. In mice, chronic VL is successfully established after intravenous or intradermal inoculation (45). Disseminated granulomas with parasitized macrophages are found during infection, especially in the liver and spleen of susceptible (BALB/c, C57BL/6) and resistant $(\mathrm{C} 3 \mathrm{H} . \mathrm{HeJ}, \mathrm{CBA}, \mathrm{DBA} / 2)$ mouse strains infected with $L$. donovani or $L$. infantum (29). Mice infected with $L$. donovani have been widely studied, but this model does not reproduce the features of active human VL (46). In mice, there is an early increase of parasite burden, but over the course of 4-8 weeks the infection spontaneously declines when an antiLeishmania cellular immune response, with the participation of both $\mathrm{CD}^{+}$and $\mathrm{CD} 8^{+} \mathrm{T}$ cells, is able to control the infection. This control is mediated by IFN- $\gamma$ production by splenic T cells, which are driven towards a Th1 phenotype by IL-12 (47-49). Interestingly, in endemic areas, there are significant numbers of individuals with subclinical infection associated with the development of antigenspecific $\mathrm{T}$ cell responses, IFN- $\gamma$ production and resistance to visceral infection (50). Therefore, the $L$. donovani murine model seems to reflect the early parasite replication followed by immunological control and subclinical infection in human disease. However, there is still no murine model to study the progressive disease observed in human VL.

Vaccine studies with the murine models of $\mathrm{VL}$ are not as developed as the models used for $\mathrm{CL}$. The levels of protection found in vaccine studies of murine $\mathrm{VL}$ are significantly lower when compared with murine CL models $(46,51-59)$. It is unclear whether the low level of protection observed in VL vaccine studies is due to the higher infective dose, the routes of challenge used (intravenous versus subcutaneous or intradermal), the requirement for immunological effector control mechanisms that are not adequately induced by vaccination, or a combination of all factors (45). In order to address these variables and to improve the murine model of $\mathrm{VL}$, an intradermal model of infection was explored and chronic VL was successfully established in susceptible mice. In this model, the course of infection was associated with parasite clearance in the liver and skin and persistence of Leishmania in the spleen and draining lymph node. Interestingly, a similar finding is also observed in subclinical canine and human VL. The site-specific parasite clearance or persistence is strongly correlated with distinct localized immune responses. Mice vaccinated with $L$. infantum D-13 (p80) antigen and challenged intradermally showed higher levels of protection when compared to a low-dose intravenous infection model (45). In contrast, the hamster model for VL mimics several aspects of human disease, such as hepatosplenomegaly, pancytopenia, progressive cachexia, hypergammaglobulinemia, and suppression of a T-cell proliferative response to parasite antigens (60-62). Hamster and human VL have been related to the inability of infected antigen-presenting cells to stimulate specific $T$ cells $(61,62)$. This is supported by the observation that, in spite of the production of Th1 cytokines (IL-2, IFN- $\gamma$ and $\mathrm{TNF}-\alpha)$ in the liver, spleen and bone marrow, the animal cannot control parasite replication, suggesting an impairment of macrophage function. Disease progression of hamster VL was associated with lack of nitric oxide (NO) due to the absence of NO synthase activity despite a strong IFN- $\gamma$ production in the liver and spleen during the course of infection (63). Although the hamster VL model can be closely related to human VL in many clinical and pathological aspects, it is severely restricted by the limited availability of tools to dissect immune responses and mechanisms. Progressive disease in hamsters has been mostly achieved by intravenous, intracardiac or intraperitoneal injection of a large number of parasites (63-65). Gomes et al. (66) showed the fatal outcome of VL in 3- to 4-month-old naive hamsters after intradermal injection of Leishmania in the ear together with sand fly saliva. Hamsters developed classical signs of VL, culminating in a fatal outcome 5-6 months post-infection. Although saliva had no effect on the course of infection in this model, a novel 11-kDa protein (LJM19) from L. longipalpis saliva protected hamsters against the fatal outcome of VL. 
LJM19-vaccinated hamsters maintained a low parasite load correlating with high IFN- $\gamma$, TGF- $\beta$ and $\mathrm{NO}$ in the spleen and liver up to 5 months post-infection. Importantly, a DTH response with high expression of IFN- $\gamma$ was also observed in the skin of LJM19-immunized hamsters $48 \mathrm{~h}$ after exposure to uninfected $L$. longipalpis bites. The induction of IFN- $\gamma$ at the bite site could partly explain the protection observed in the spleen and liver of LJM19-immunized hamsters through direct parasite killing and/or priming of anti-Leishmania immunity (66).

The dog is considered to be the main reservoir of $L$. chagasi in Latin America. Disease control is based on culling of Leishmania-positive animals, which has generated ethical and social discussions. A vaccine capable of preventing canine visceral leishmaniasis (CVL) will be a good alternative for epidemiological control of all VL (67). In order to better understand leishmaniasis in dogs, several studies have investigated different inoculation routes and different parasite quantities of either amastigotes or promastigotes. Although those studies reproduced some features of naturally acquired CVL, in nature, transmission occurs after an infected sand fly takes a blood meal from the skin of the animal. Some of the naturally infected dogs will remain asymptomatic, but others will develop a progressive disease with lymphoadenopathy, weight loss, anemia, hypergammaglobulinemia and dermatitis ultimately resulting in death $(68,69)$. Thus, a proper canine model for vaccine research should reproduce the immunopathological features of the natural disease. The dermis would be the preferential site of vaccine inoculation or infection challenge since intradermal inoculation mimics the delivery of antigen and/or infection by sand flies.

Several antigens, including live or killed parasites, purified Leishmania fractions and defined recombinant proteins, live recombinant bacteria expressing parasite antigens, antigen-encoding DNA plasmids and recombinant salivary proteins, have been identified and tested as potential vaccine candidates against CVL $(32,66,70-73)$.

The use of dogs or rodents as models for a $V L$ vaccine selection has allowed the development of two CVL vaccines that are commercially available: Leishmune

\section{References}

1. Adams EJ, Cooper S, Thomson G, Parham P. Common chimpanzees have greater diversity than humans at two of the three highly polymorphic MHC class I genes. Immunogenetics 2000; 51: 410-424, doi: 10.1007/s002510050639.

2. Muchmore EA. Chimpanzee models for human disease and immunobiology. Immunol Rev 2001; 183: 86-93, doi: 10.1034/j.1600-065x.2001.1830107.x.

3. Miller LH, Good MF, Milon G. Malaria pathogenesis. Science 1994; 264: 1878-1883, doi: 10.1126/science.8009217.

4. Clyde DF. Immunization of man against falciparum and vivax malaria by use of attenuated sporozoites. Am J Trop and Leish-Tec. Leishmune vaccine is a prophylactic vaccine against $C V L$ and was the first licensed secondgeneration vaccine against leishmaniasis (71). This vaccine consists of the fucose mannose ligand isolated from Leishmania donovani and saponin as an adjuvant (74). Leishmune showed efficacy in mice, hamsters and dogs and also protected dogs living in an endemic VL area in Brazil (75-77).

Leish-Tec was the second vaccine commercially developed against CVL and is composed of an amastigote-specific antigen (A2) and saponin as an adjuvant also induced protection in the field $(76,77)$. These results support the importance of the dog as a model and target for vaccines against $V L$, an important approach to reducing disease incidence in dogs and ultimately reducing human infections.

\section{Final remarks}

When vaccines are being evaluated, an important point of concern is the identification of correlates of protection obtained from animal models. The selection of the right animal model, the pathogen-model combination, sometimes needs to be adapted to represent human infection. The immune response to a given pathogen may also vary from one animal strain to the other and the results of the administration of a potential vaccine candidate in an animal model may not be the same as results of human clinical and endemic area trials. Therefore, biomarkers obtained with animal models adapted for vector-borne parasitic diseases should always be carefully evaluated and validation using natural transmission of parasites is of the utmost importance since an infected bite is the final challenge that any vaccine will face in an endemic area.

\section{Acknowledgments}

We thank Dr. Theolis Bessa (Centro de Pesquisas Gonçalo Moniz, Fiocruz, Brazil) for a critical review of this manuscript. We also thank Dr. Ryan Jochim (Walter Reed Army Institute of Research, Entomology Branch, USA) for critical and English review of this manuscript.
Med Hyg 1975; 24: 397-401.

5. Nussenzweig RS, Vanderberg J, Most H, Orton C Protective immunity produced by the injection of $\mathrm{x}$-irradiated sporozoites of Plasmodium berghei. Nature 1967; 216: 160162, doi: 10.1038/216160a0.

6. Siddiqui WA, Taylor DW, Kan SC, Kramer K, RichmondCrum SM, Kotani S, et al. Vaccination of experimental monkeys against Plasmodium falciparum: a possible safe adjuvant. Science 1978; 201: 1237-1239, doi: 10.1126/ science.99814.

7. Vanderberg JP. Reflections on early malaria vaccine 
studies, the first successful human malaria vaccination, and beyond. Vaccine 2009; 27: 2-9, doi: 10.1016/j.vaccine.2008.10.028.

8. Good MF. The hope but challenge for developing a vaccine that might control malaria. Eur J Immunol 2009; 39: 939943, doi: 10.1002/eji.200939390.

9. Wykes MN, Good MF. What have we learnt from mouse models for the study of malaria? Eur J Immunol 2009; 39: 2004-2007, doi: 10.1002/eji.200939552.

10. Vinvke $\mathrm{IH}$. Experimental transmission of Plasmodium berghei. Indian J Malariol 1954; 8: 257-262.

11. Carlton JM, Hayton K, Cravo PV, Walliker D. Of mice and malaria mutants: unravelling the genetics of drug resistance using rodent malaria models. Trends Parasitol 2001; 17: 236-242, doi: 10.1016/S1471-4922(01)01899-2.

12. Rodrigues MM, Cordey AS, Arreaza G, Corradin G, Romero $\mathrm{P}$, Maryanski JL, et al. CD8 ${ }^{+}$cytolytic $\mathrm{T}$ cell clones derived against the Plasmodium yoelii circumsporozoite protein protect against malaria. Int Immunol 1991; 3: 579-585, doi: 10.1093/intimm/3.6.579.

13. Rodrigues $M$, Nussenzweig RS, Zavala $F$. The relative contribution of antibodies, $\mathrm{CD}^{+}$and $\mathrm{CD}^{+} \mathrm{T}$ cells to sporozoite-induced protection against malaria. Immunology 1993; 80: 1-5.

14. Tsuji M, Romero P, Nussenzweig RS, Zavala F. CD4 ${ }^{+}$ cytolytic $\mathrm{T}$ cell clone confers protection against murine malaria. J Exp Med 1990; 172: 1353-1357, doi: 10.1084/ jem.172.5.1353.

15. Tsuji M. A retrospective evaluation of the role of $\mathrm{T}$ cells in the development of malaria vaccine. Exp Parasitol 2010; 126: 421-425, doi: 10.1016/j.exppara.2009.11.009.

16. Stowers AW, Cioce V, Shimp RL, Lawson M, Hui G, Muratova $\mathrm{O}$, et al. Efficacy of two alternate vaccines based on Plasmodium falciparum merozoite surface protein 1 in an Aotus challenge trial. Infect Immun 2001; 69: 1536-1546, doi: 10.1128/IAI.69.3.1536-1546.2001.

17. Aikawa M, Brown A, Smith CD, Tegoshi T, Howard RJ, Hasler $\mathrm{TH}$, et al. A primate model for human cerebral malaria: Plasmodium coatneyi-infected rhesus monkeys. Am J Trop Med Hyg 1992; 46: 391-397.

18. Mclntosh RS, Shi J, Jennings RM, Chappel JC, de KoningWard TF, Smith T, et al. The importance of human FcgammaRI in mediating protection to malaria. PLoS Pathog 2007; 3: e72, doi: 10.1371/journal.ppat.0030072.

19. Vaughan AM, Kappe SH, Ploss A, Mikolajczak SA. Development of humanized mouse models to study human malaria parasite infection. Future Microbiol 2012; 7: 657665, doi: 10.2217/fmb.12.27.

20. Fonseca L, Seixas E, Butcher G, Langhorne J. Cytokine responses of $\mathrm{CD}^{+}{ }^{+} \mathrm{T}$ cells during a Plasmodium chabaudi chabaudi (ER) blood-stage infection in mice initiated by the natural route of infection. Malar J 2007; 6: 77, doi: 10.1186/ 1475-2875-6-77.

21. Leitner WW, Bergmann-Leitner ES, Angov E. Comparison of Plasmodium berghei challenge models for the evaluation of pre-erythrocytic malaria vaccines and their effect on perceived vaccine efficacy. Malar J 2010; 9: 145, doi: 10.1186/1475-2875-9-145.

22. Handman E. Leishmaniasis: current status of vaccine development. Clin Microbiol Rev 2001; 14: 229-243, doi: 10.1128/CMR.14.2.229-243.2001.
23. Duthie MS, Raman VS, Piazza FM, Reed SG. The development and clinical evaluation of second-generation leishmaniasis vaccines. Vaccine 2012; 30: 134-141, doi: 10.1016/j.vaccine.2011.11.005.

24. Titus RG, Ribeiro JM. Salivary gland lysates from the sand fly Lutzomyia longipalpis enhance Leishmania infectivity. Science 1988; 239: 1306-1308, doi: 10.1126/science. 3344436.

25. Rogers ME, Ilg T, Nikolaev AV, Ferguson MA, Bates PA. Transmission of cutaneous leishmaniasis by sand flies is enhanced by regurgitation of fPPG. Nature 2004; 430: 463467, doi: 10.1038/nature02675.

26. Belkaid Y, Kamhawi S, Modi G, Valenzuela J, Noben-Trauth $\mathrm{N}$, Rowton $\mathrm{E}$, et al. Development of a natural model of cutaneous leishmaniasis: powerful effects of vector saliva and saliva preexposure on the long-term outcome of Leishmania major infection in the mouse ear dermis. $J$ Exp Med 1998; 188: 1941-1953, doi: 10.1084/jem.188.10. 1941.

27. Sacks $D$, Noben-Trauth $N$. The immunology of susceptibility and resistance to Leishmania major in mice. Nat Rev Immunol 2002; 2: 845-858, doi: 10.1038/nri933.

28. Grimaldi G Jr.. The utility of rhesus monkey (Macaca mulatta) and other non-human primate models for preclinical testing of Leishmania candidate vaccines. Mem Inst Oswaldo Cruz 2008; 103: 629-644, doi: 10.1590/S007402762008000700002 .

29. de Oliveira Cl, Teixeira M, Gomes R, Barral A, Brodskyn C. Animal models for infectious diseases caused by parasites: leishmaniasis. Drug Discovery Today: Disease Models 2004; 1: 81-86, doi: 10.1016/j.ddmod.2004.07.005.

30. Preston PM, Dumonde DC. Experimental cutaneous Leishmaniasis. V. Protective immunity in subclinical and self-healing infection in the mouse. Clin Exp Immunol 1976; 23: $126-138$.

31. Liew FY, Li Y, Millott $\mathrm{S}$. Tumor necrosis factor-alpha synergizes with IFN-gamma in mediating killing of Leishmania major through the induction of nitric oxide. $J$ Immunol 1990; 145: 4306-4310.

32. Gomes R, Oliveira F, Teixeira C, Meneses C, Gilmore DC, Elnaiem DE, et al. Immunity to sand fly salivary protein LJM11 modulates host response to vector-transmitted Leishmania conferring ulcer-free protection. J Invest Dermatol 2012; 132: 2735-2743, doi: 10.1038/jid.2012.205.

33. Samuelson J, Lerner E, Tesh R, Titus R. A mouse model of Leishmania braziliensis braziliensis infection produced by coinjection with sand fly saliva. J Exp Med 1991; 173: 49-54, doi: $10.1084 /$ jem.173.1.49.

34. Theodos CM, Ribeiro JM, Titus RG. Analysis of enhancing effect of sand fly saliva on Leishmania infection in mice. Infect Immun 1991; 59: 1592-1598.

35. Lima HC, Titus RG. Effects of sand fly vector saliva on development of cutaneous lesions and the immune response to Leishmania braziliensis in BALB/c mice. Infect Immun 1996; 64: 5442-5445.

36. Nong YH, Titus RG, Ribeiro JM, Remold HG. Peptides encoded by the calcitonin gene inhibit macrophage function. J Immunol 1989; 143: 45-49.

37. Qureshi AA, Asahina A, Ohnuma M, Tajima M, Granstein $\mathrm{RD}$, Lerner EA. Immunomodulatory properties of maxadilan, the vasodilator peptide from sand fly salivary gland extracts. 
Am J Trop Med Hyg 1996; 54: 665-671.

38. Rogers KA, Titus RG. Immunomodulatory effects of Maxadilan and Phlebotomus papatasi sand fly salivary gland lysates on human primary in vitro immune responses. Parasite Immunol 2003; 25: 127-134, doi: 10.1046/j.13653024.2003.00623.x.

39. Valenzuela JG, Belkaid Y, Garfield MK, Mendez S, Kamhawi S, Rowton ED, et al. Toward a defined antiLeishmania vaccine targeting vector antigens: characterization of a protective salivary protein. J Exp Med 2001; 194: 331-342, doi: 10.1084/jem.194.3.331.

40. Morris RV, Shoemaker CB, David JR, Lanzaro GC, Titus RG. Sandfly maxadilan exacerbates infection with Leishmania major and vaccinating against it protects against $L$. major infection. J Immunol 2001; 167: 5226-5230.

41. Kamhawi S, Belkaid Y, Modi G, Rowton E, Sacks D. Protection against cutaneous leishmaniasis resulting from bites of uninfected sand flies. Science 2000; 290: 13511354, doi: 10.1126/science.290.5495.1351.

42. Rogers ME, Sizova OV, Ferguson MA, Nikolaev AV, Bates PA. Synthetic glycovaccine protects against the bite of Leishmania-infected sand flies. J Infect Dis 2006; 194: 512518, doi: $10.1086 / 505584$

43. Peters NC, Kimblin N, Secundino N, Kamhawi S, Lawyer P, Sacks DL. Vector transmission of Leishmania abrogates vaccine-induced protective immunity. PLoS Pathog 2009; 5: e1000484, doi: 10.1371/journal.ppat.1000484.

44. Gomes R, Teixeira C, Oliveira F, Lawyer PG, Elnaiem DE, Meneses C, et al. KSAC, a defined Leishmania antigen, plus adjuvant protects against the virulence of $L$. major transmitted by its natural vector Phlebotomus duboscqi. PLoS Negl Trop Dis 2012; 6: e1610, doi: 10.1371/journal. pntd.0001610.

45. Ahmed S, Colmenares M, Soong L, Goldsmith-Pestana K, Munstermann L, Molina R, et al. Intradermal infection model for pathogenesis and vaccine studies of murine visceral leishmaniasis. Infect Immun 2003; 71: 401-410, doi: 10.1128/IAI.71.1.401-410.2003.

46. Melby PC, Yang J, Zhao W, Perez LE, Cheng J. Leishmania donovani $\mathrm{p} 36(\mathrm{LACK})$ DNA vaccine is highly immunogenic but not protective against experimental visceral leishmaniasis. Infect Immun 2001; 69: 4719-4725, doi: 10.1128/ IAI.69.8.4719-4725.2001.

47. Squires KE, Schreiber RD, McElrath MJ, Rubin BY, Anderson SL, Murray HW. Experimental visceral leishmaniasis: role of endogenous IFN-gamma in host defense and tissue granulomatous response. J Immunol 1989; 143: 4244-4249.

48. Murray HW, Masur H, Keithly JS. Cell-mediated immune response in experimental visceral leishmaniasis. I. Correlation between resistance to Leishmania donovani and lymphokine-generating capacity. J Immunol 1982; 129: 344-350.

49. Murray HW. Endogenous interleukin-12 regulates acquired resistance in experimental visceral leishmaniasis. $J$ Infect Dis 1997; 175: 1477-1479, doi: 10.1086/516482.

50. Badaro R, Jones TC, Carvalho EM, Sampaio D, Reed SG, Barral A, et al. New perspectives on a subclinical form of visceral leishmaniasis. J Infect Dis 1986; 154: 1003-1011, doi: 10.1093/infdis/154.6.1003.

51. Champsi J, Mahon-Pratt D. Membrane glycoprotein M-2 protects against Leishmania amazonensis infection. Infect Immun 1988; 56: 3272-3279.

52. Jardim A, Alexander J, Teh HS, Ou D, Olafson RW. Immunoprotective Leishmania major synthetic T cell epitopes. J Exp Med 1990; 172: 645-648, doi: 10.1084/jem. 172.2.645.

53. Rachamim N, Jaffe CL. Pure protein from Leishmania donovani protects mice against both cutaneous and visceral leishmaniasis. J Immunol 1993; 150: 2322-2331.

54. Soong L, Duboise SM, Kima P, Mahon-Pratt D. Leishmania pifanoi amastigote antigens protect mice against cutaneous leishmaniasis. Infect Immun 1995; 63: 3559-3566.

55. McSorley SJ, Xu D, Liew FY. Vaccine efficacy of Salmonella strains expressing glycoprotein 63 with different promoters. Infect Immun 1997; 65: 171-178.

56. Gurunathan S, Sacks DL, Brown DR, Reiner SL, Charest H, Glaichenhaus N, et al. Vaccination with DNA encoding the immunodominant LACK parasite antigen confers protective immunity to mice infected with Leishmania major. J Exp Med 1997; 186: 1137-1147, doi: 10.1084/jem.186.7.1137.

57. Stager S, Smith DF, Kaye PM. Immunization with a recombinant stage-regulated surface protein from Leishmania donovani induces protection against visceral leishmaniasis. J Immunol 2000; 165: 7064-7071.

58. Ghosh A, Labrecque S, Matlashewski G. Protection against Leishmania donovani infection by DNA vaccination: increased DNA vaccination efficiency through inhibiting the cellular p53 response. Vaccine 2001; 19: 3169-3178, doi: 10.1016/S0264-410X(01)00023-8.

59. Paraguai de Souza E, Bernardo RR, Palatnik M, Palatnik de Sousa CB. Vaccination of BALB/C mice against experimental visceral leishmaniasis with the GP36 glycoprotein antigen of Leishmania donovani. Vaccine 2001; 19: 31043115, doi: 10.1016/S0264-410X(01)00031-7.

60. Bunn-Moreno MM, Madeira ED, Miller K, Menezes JA, Campos-Neto A. Hypergammaglobulinaemia in Leishmania donovani infected hamsters: possible association with a polyclonal activator of $B$ cells and with suppression of $T$ cell function. Clin Exp Immunol 1985; 59: 427-434.

61. Fruth U, Solioz N, Louis JA. Leishmania major interferes with antigen presentation by infected macrophages. $J$ Immunol 1993; 150: 1857-1864.

62. Rodrigues Junior V, da Silva JS, Campos-Neto A. Selective inability of spleen antigen presenting cells from Leishmania donovani infected hamsters to mediate specific $\mathrm{T}$ cell proliferation to parasite antigens. Parasite Immunol 1992; 14: 49-58, doi: 10.1111/j.1365-3024.1992.tb00005.x.

63. Melby PC, Yang YZ, Cheng J, Zhao W. Regional differences in the cellular immune response to experimental cutaneous or visceral infection with Leishmania donovani. Infect Immun 1998; 66: 18-27.

64. Bories C, Coffin C, Mathieu D, Bories PN, Scherman E, Rivollet $\mathrm{D}$, et al. Lack of a nitric-oxide response during the course of Leishmania infantum infection in the golden hamster (Mesocricetus auratus), with or without treatment with liposomal amphotericin B. Ann Trop Med Parasitol 1998; 92: 685-692, doi: 10.1080/00034989859140.

65. Basu R, Bhaumik S, Basu JM, Naskar K, De T, Roy S. Kinetoplastid membrane protein-11 DNA vaccination induces complete protection against both pentavalent antimonial-sensitive and -resistant strains of Leishmania 
donovani that correlates with inducible nitric oxide synthase activity and IL-4 generation: evidence for mixed Th1- and Th2-like responses in visceral leishmaniasis. J Immunol 2005; 174: 7160-7171.

66. Gomes R, Teixeira C, Teixeira MJ, Oliveira F, Menezes MJ, Silva C, et al. Immunity to a salivary protein of a sand fly vector protects against the fatal outcome of visceral leishmaniasis in a hamster model. Proc Natl Acad Sci U S A 2008; 105: 7845-7850, doi: 10.1073/pnas.0712153105.

67. Barbieri CL. Immunology of canine leishmaniasis. Parasite Immunol 2006; 28: 329-337, doi: 10.1111/j.1365-3024. 2006.00840.x

68. Abranches P, Santos-Gomes G, Rachamim N, Campino L, Schnur LF, Jaffe CL. An experimental model for canine visceral leishmaniasis. Parasite Immunol 1991; 13: 537 550, doi: 10.1111/j.1365-3024.1991.tb00550.x.

69. Paranhos-Silva M, Oliveira GG, Reis EA, de Menezes RM, Fernandes $\mathrm{O}$, Sherlock I, et al. A follow-up of Beagle dogs intradermally infected with Leishmania chagasi in the presence or absence of sand fly saliva. Vet Parasitol 2003; 114: 97-111, doi: 10.1016/S0304-4017(03)00132-8.

70. Gradoni L. An update on antileishmanial vaccine candidates and prospects for a canine Leishmania vaccine. Vet Parasitol 2001; 100: 87-103, doi: 10.1016/S0304-4017(01) 00486-1.

71. Palatnik-de-Sousa CB. Vaccines for leishmaniasis in the fore coming 25 years. Vaccine 2008; 26: 1709-1724, doi: 10.1016/j.vaccine.2008.01.023.
72. Palatnik-de-Sousa CB. Vaccines for canine leishmaniasis Front Immunol 2012; 3: 69.

73. Collin N, Gomes R, Teixeira C, Cheng L, Laughinghouse A Ward JM, et al. Sand fly salivary proteins induce strong cellular immunity in a natural reservoir of visceral leishmaniasis with adverse consequences for Leishmania. PLoS Pathog 2009; 5: e1000441, doi: 10.1371/journal.ppat. 1000441.

74. Gamboa-Leon R, Paraguai de SE, Borja-Cabrera GP, Santos FN, Myashiro LM, Pinheiro RO, et al. Immunotherapy against visceral leishmaniasis with the nucleoside hydrolase-DNA vaccine of Leishmania donovani. Vaccine 2006; 24: 4863-4873, doi: 10.1016/j.vaccine.2006.03.005.

75. Palatnik-de-Sousa CB, Paraguai-de-Souza E, Gomes EM, Borojevic R. Experimental murine Leishmania donovani infection: immunoprotection by the fucose-mannose ligand (FML). Braz J Med Biol Res 1994; 27: 547-551.

76. Fernandes AP, Costa MM, Coelho EA, Michalick MS, de Freitas E, Melo MN, et al. Protective immunity against challenge with Leishmania (Leishmania) chagasi in beagle dogs vaccinated with recombinant $A 2$ protein. Vaccine 2008; 26: 5888-5895, doi: 10.1016/j.vaccine.2008.05.095.

77. Fernandes AP, Coelho EA, Machado-Coelho GL, Grimaldi G Jr., Gazzinelli RT. Making an anti-amastigote vaccine for visceral leishmaniasis: rational, update and perspectives. Curr Opin Microbiol 2012; 15: 476-485, doi: 10.1016/ j.mib.2012.05.002. 